\title{
Creation of Innovated Macrocyclic Sulfazan-Formazan Compounds and Linear Sulfazan-Formazan for the first Time Globally with their Assay as Antifungal
}

\author{
Nagham Mahmood Aljamali* \\ Organic Chemistry, Synthetic Chemistry Field, Iraq \\ *Corresponding author: Nagham Mahmood Aljamali, Professor, Ph. D, Organic Chemistry, Synthetic Chemistry Field, Iraq
}

ARTICLE INFO

Received: December 01, 2021

Published: 幽 December 09, 2021

Citation: Nagham Mahmood Aljamali. Creation of Innovated Macrocyclic Sulfazan-Formazan Compounds and Linear Sulfazan-Formazan for the first Time Globally with their Assay as Antifungal. Biomed J Sci \& Tech Res 40(3)-2021. BJSTR. MS.ID.006453.

\begin{abstract}
Sulfazan compounds were invented for the first time globally by researcher Dr. Nagham Aljamali in 2019 was named as sulfazan compounds, then it was developed into cyclic sulfazan and cyclic formazan, which was also first prepared in April 2021 in this current study., which prepared by using various conditions and different basic medium like (Pyridine, Pipridine, 5 \% Sodium hydroxide, Triethyl amine,...) .,Several technical spectroscopic studies have been used to prove their chemical structures which gave clear evidence of its chemical structures via many technical instruments like (FT IR-Spectra , 1H.NMR-Spectra, Mass-Spectra)., Melting points, other studies represented by evolution them as antifungal compounds.
\end{abstract}

Keywords: Sulfazan; Cyclic Sulfazan; Cyclic Formazan; Formazan; Imine; Schiff Base; Azo; Antifungal; Aldamine; Anil; Triazole; Oxadiazole

\section{Introduction}

Sulfazan compounds were invented for the first time globally by researcher Prof. Dr. Nagham Aljamali in 2019 was named as sulfazan compounds [1-3]. The researcher also developed and devised the basics of their preparation methods, the conditions of each method, their interactions, the reaction medium, and their naming. Then she developed them into cyclic sulfazan and cyclic formazan compounds [4-7] that were also prepared for the first time in April 2021 in this current study and also developed and established methods for their preparation and reaction conditions, and the current research included the preparation of innovative types of cyclic sulfazan compounds, linear sulfazan, cyclic formazan and linear formazan.

\section{Sulfazan Compounds}

Are organic sulfur compounds that were prepared in researches [1-3] as first time by Dr. Nagham Aljamali in 2019 from several reactions that include the reaction of the coupling of basic mercapto and thiol compounds with azo compounds using one of the conditions (pyridine, piperidine, triethylamine,...) [7-9] and its chemical structure is (Ar-N=N-S-R).

\section{Formazan Compounds}

Are a class of organic compounds of importance in organic chemistry because it contains two highly effective groups $(-\mathrm{N}=\mathrm{N}=\mathrm{C}-\mathrm{N}-)$ or $(-\mathrm{N}=\mathrm{N}-\mathrm{C}-\mathrm{N}-\mathrm{NH}-)$ in several fields of chemistry [8-14], especially in coordination chemistry $[15,16]$, as a ligand because they contain free electrons and donor atoms to coordinate with ions to form complexes [17-19] and types of them as anticancer [20-22].

\section{Cyclic Formazan and Cyclic Sulfazan}

These compounds invented by the researcher Dr. Nagham Aljamali in April 2021 for the first time [4,5]. Initial foundations and methods for preparing these compounds were established, 
and to determine the conditions [4-8] of their interaction and the auxiliary factors used to prepare them. They were considered among the organic compounds of importance in organic chemistry because they contain two highly effective groups in several fields of chemistry, especially in coordination chemistry, as a ligands because they contain doublets. Free electrons and donor atoms to coordinate with ions to form complexes [23-28]. Formazan also enter many anti-bacterial [29-33] and anti-fungal compounds [34$37]$ and types of cancer [38,39], especially breast and laryngeal cancers, as anti-bacterial and anti-fungal, and other studies $[40,41]$.

\section{Instruments and Experimental Part}

All melting points were uncorrected and dignified on an electrothermal apparatus (Switzerland) in an open capillary tube. FT.IR spectra were detailed on Fourier transform infrared spectrometer (FT-IR) in( FT-IR- 3600) infrared spectrometer via employing $\mathrm{KBr}$
Pellet technique., 1H.NMR spectra were recorded in DMSO-d6 as solvent using (TMS) as internal standard and chemical shifts are expressed as ( $\delta \mathrm{ppm})$., also Mass- Spectra for some of them other studies like evaluation against types of fungi).

\section{Procedures}

\section{Preparation of Compounds $\{1,2\}$}

Cysteine (0.01 mole) was dissolved in $(30 \mathrm{ml})$ absolute ethanol with semicarbazide( 0.01 mole) with refluxing for (10hrs) in presence of phosphoric acid as closing agent, according to procedures [6-9], the product filtered ,dried ,recrystallized to yield Oxadiazole amine Derivative-Compound [1], which reacted $(0.01$ mole) in $(50 \mathrm{ml})$ absolute ethanol with $\mathrm{p}$-iodobenzaldehyde $(0.02$ mole) with refluxing for ( $3 \mathrm{hrs}$ ) in presence of (3 drops of glacial acetic acid), according to procedure [6- 9], the product filtered ,dried ,recrystallized to yield Aldamine -Compound [2] (Figure 1).

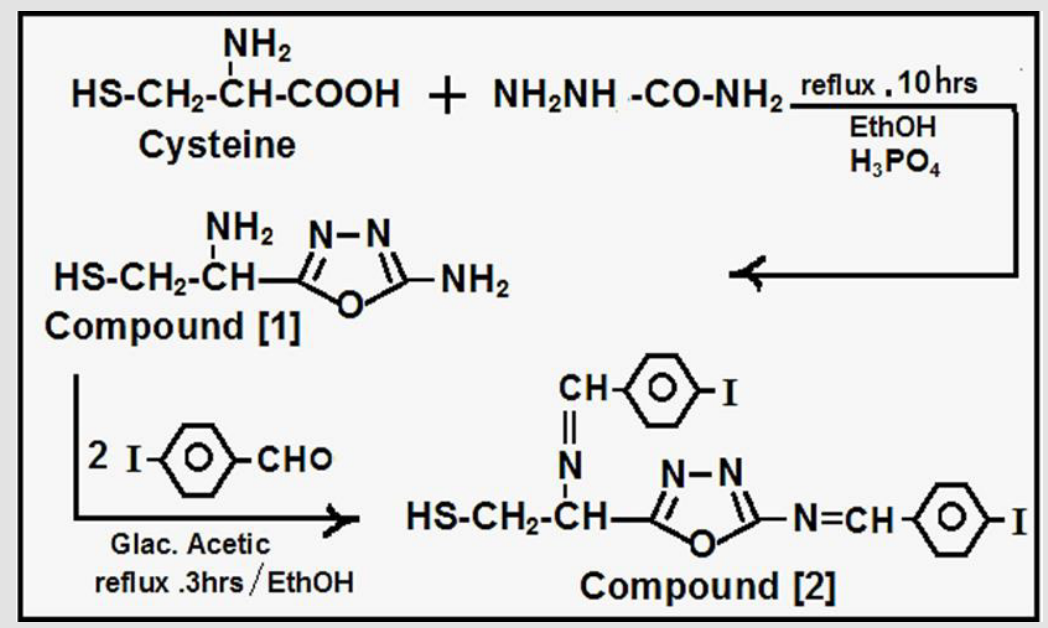

Figure 1: Synthesis of Compounds $\{1,2\}$

\section{Creation of Inventive Macrocyclic Sulfazan-Formazan Compound $\{3\}$}

Aldamine compound [2]was (0.01 mole) dissolved in basic solution from (Piprydine) then reacted with $(0.02$ mole $)$ of diazo salt of o- phenyl diamine via many steps in basic solution to formation invented Macrocyclic Sulfazan-Formazan after (48hrs), the product filtered ,dried, washed by distilled water, recrystallized to yield Invented Macrocyclic Sulfazan- Formazan [3] by following literatures [1-5].

\section{Creation of Inventive Macrocyclic Sulfazan-Formazan Compound $\{4\}$}

Macrocyclic Sulfazan-Formazan Compound\{3\} refluxed $[0.01$ mole) for (4hrs) in $(40 \mathrm{ml})$ absolute ethanol in presence of cupper acetate $(0.92 \mathrm{gm})$ in closing step of amine group in ortho- position from azo- group to formation Triazole derivative according to procedures [6,7] , the product filtered ,dried ,recrystallized to yield Invented Macrocyclic Sulfazan-formazan of Triazole derivative [4], according to procedures [6-10] (Figure 2). 


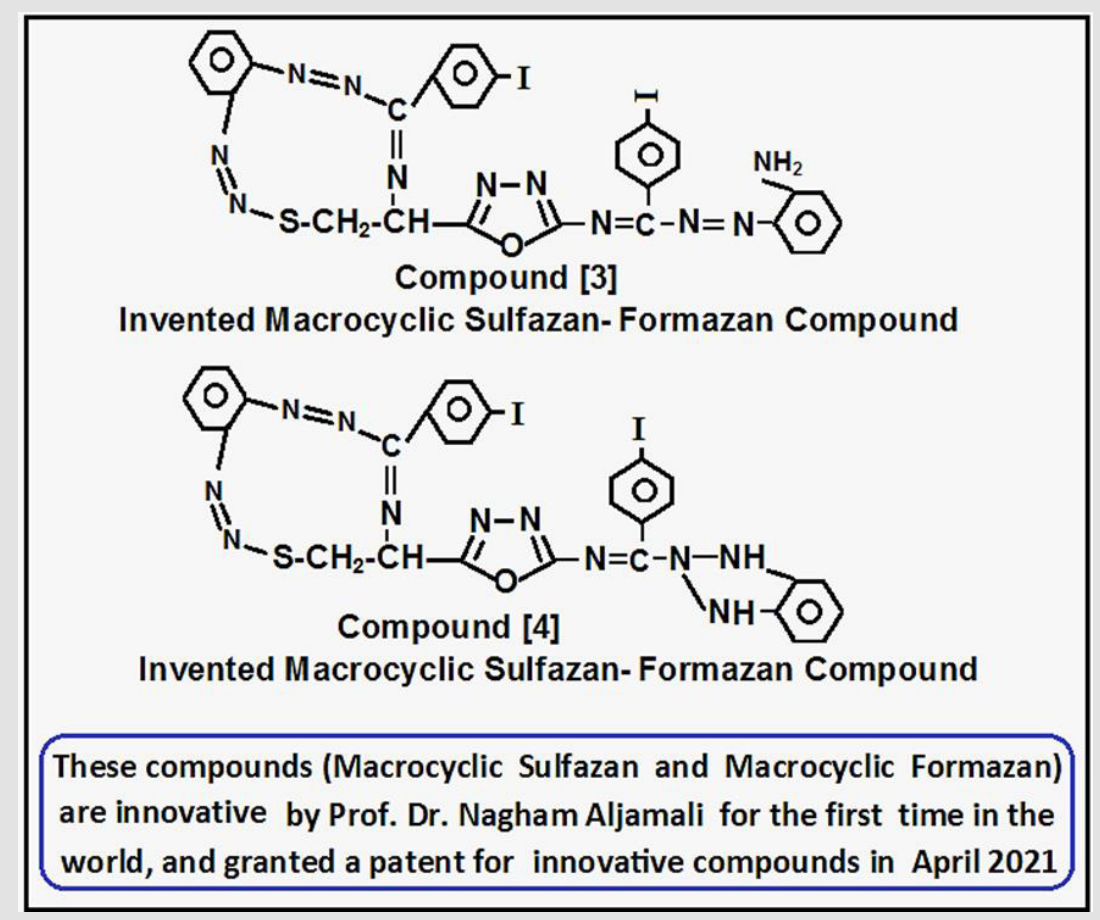

Figure 2: Creation of Invented Macrocyclic Sulfazan- Formazan Compounds $\{3,4\}$.

\section{Creation of Inventive Linear Sulfazan- Formazan Compound $\{5\}$}

Aldamine Compound [2] about ( 0.01 mole) was reacted basic solution (Pyridine) via many steps in coupling reaction with $(0.03$ mole) of p-bromophenyl diazonium salt in basic medium, after (10hrs), the product filtered ,dried ,washed by distilled water, recrystallized to yield Invented Sulfazan- Formazan [5], according to procedures [1-5].

\section{Creation of Inventive Linear Sulfazan- Formazan Compound $\{6\}$}

Aldamine Compound [2] about (0.01 mole) was reacted basic solution (Pipyridine) via many steps in coupling reaction with (0.03 mole) of p-methoxyphenyl diazonium salt in basic medium, after (10hrs), the product filtered,dried,washed by distilled water, recrystallized to yield Invented Sulfazan- Formazan [6], according to procedures [1-5] (Figure 3).

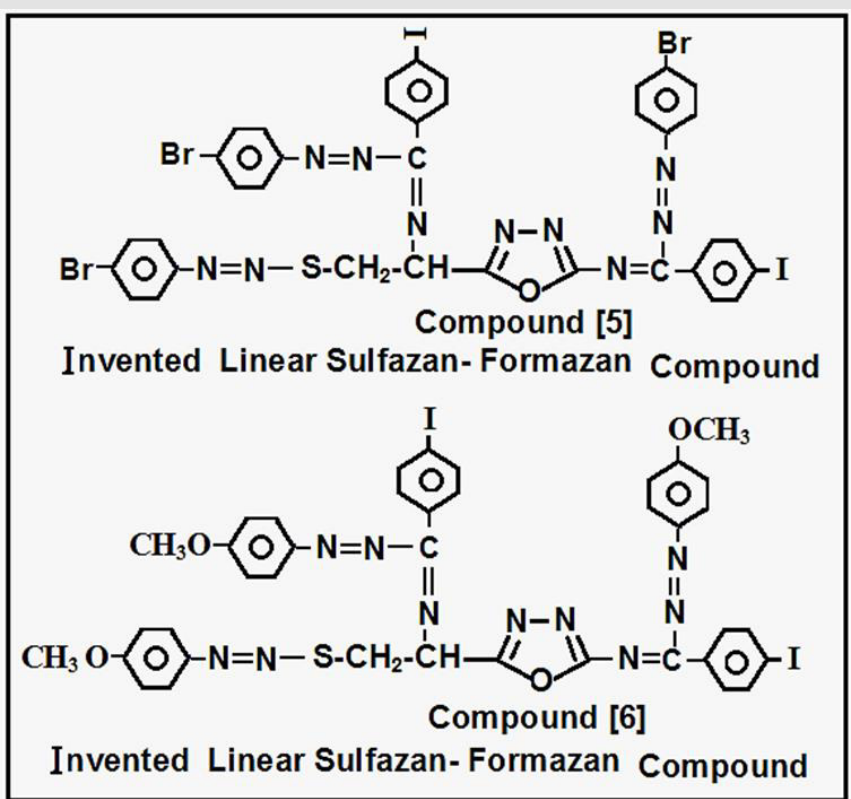

Figure 3: Creation of Invented Sulfazan- Formazan Compounds $\{5,6\}$. 


\section{Results and Discussion}

In recently study, various of Invented Macrocyclic SulfazanFormazan Compounds and linear Sulfazan- Formazan have been created in same procedure that followed and invented [1-5] by Dr. Nagham in year 2019 that got a patent to invention of Macrocyclic Sulfazan-Formazan compounds, then several studies were carried out to improve these innovative compounds by the using of spectral identification like : 1H.NMR spectra, FT.IR- Spectra, Mass- Spectra., other studies represented by (Melting points, evaluation against types of fungi)., all the results are revealed in Tables and figures.

\section{Spectral Analysis}

FT.IR- Spectral Evidence of Invented Macrocyclic SulfazanFormazan Compounds and Linear Sulfazan-Formazan Compounds : The first characterization of innovative compounds by shifting of frequencies of Aldamine group $(\mathrm{CH}=\mathrm{N})$ in starting compounds (Imine compounds) that were about at (1612) $\mathrm{Cm}^{-1}$ in starting compounds (imine compounds) were shifted to (1627) $\mathrm{Cm}^{-1}$ for $(-\mathrm{C}=\mathrm{N}-)$ due to formation of Macrocyclic Formazan and band at (1310) $\mathrm{Cm}^{-1}$ due to (-S-N=N-) to Sulfazan group in Macrocyclic Sulfazan compound, also appearance of three bands due to partitions of azo group of Sulfazan and Formazan in Macrocycle $(-\mathrm{N}=\mathrm{N}-)$ are $(1423,1455,1490) \mathrm{Cm}^{-1}$ for $(-\mathrm{N}=\mathrm{N}-\mathrm{S}-)-$ Sulfazan and $(-\mathrm{N}=\mathrm{N}-\mathrm{C}-)$-Formazan in compound $\{3\}$., While in compound [4] appeared band at (3298) $\mathrm{Cm}^{-1}$ due to (NH) in Triazole ring as a result of ring closure that resulted from closing of amine group in ortho- position with Azo group to formation Triazole ring, besides to appearance of bands at (1629) $\mathrm{Cm}^{-1}$ for $(-\mathrm{C}=\mathrm{N}-)$ due to formation of Macrocyclic Formazan and band at (1304) $\mathrm{Cm}^{-1}$ due to $(-\mathrm{S}-\mathrm{N}=\mathrm{N}-$ ) to Sulfazan group in Macrocyclic Sulfazan compound , also appearance of three bands due to partitions of azo group of Sulfazan and Formazan in Macrocycle $(-\mathrm{N}=\mathrm{N}-)$ are $(1432,1456$, 1498) $\mathrm{Cm}^{-1}$ for (-N=N-S-)-Sulfazan and (-N=N-C-)-Formazan., and other compound like this., all frequencies explained according to reference [35].

\section{H.NMR- Spectral Evidence of Invented Macrocyclic Sulfazan-Formazan Compounds and Linear Sulfazan- Formazan Compounds}

The second characterization of innovative compounds by disappearance of peak for imine group $(\mathrm{CH}=\mathrm{N})$ in starting compound (Aldamine compound) that were at $\delta$ (8.27) in Compound $\{2\}$ (starting compound) due to formation of $(\mathrm{N}=\mathrm{C}-\mathrm{N}=\mathrm{N})$ for (Formazan and Sulfazan groups) in created compounds, also in compound [4] appeared peak at $\delta(5.35)$ due to $(\mathrm{NH})$ in Triazole ring as a result of ring closure that resulted from closing of amine group in ortho- position with Azo group to formation Triazole ring, all peaks explained according to reference [35].

\section{Mass- Spectral Evidence of Invented Macrocyclic Sulfazan-Formazan Compounds and Linear Sulfazan- Formazan Compounds}

The third characterization of inventive compounds by partition of innovative cyclic compounds via appearance of fragments in spectra in Figures 4-6.

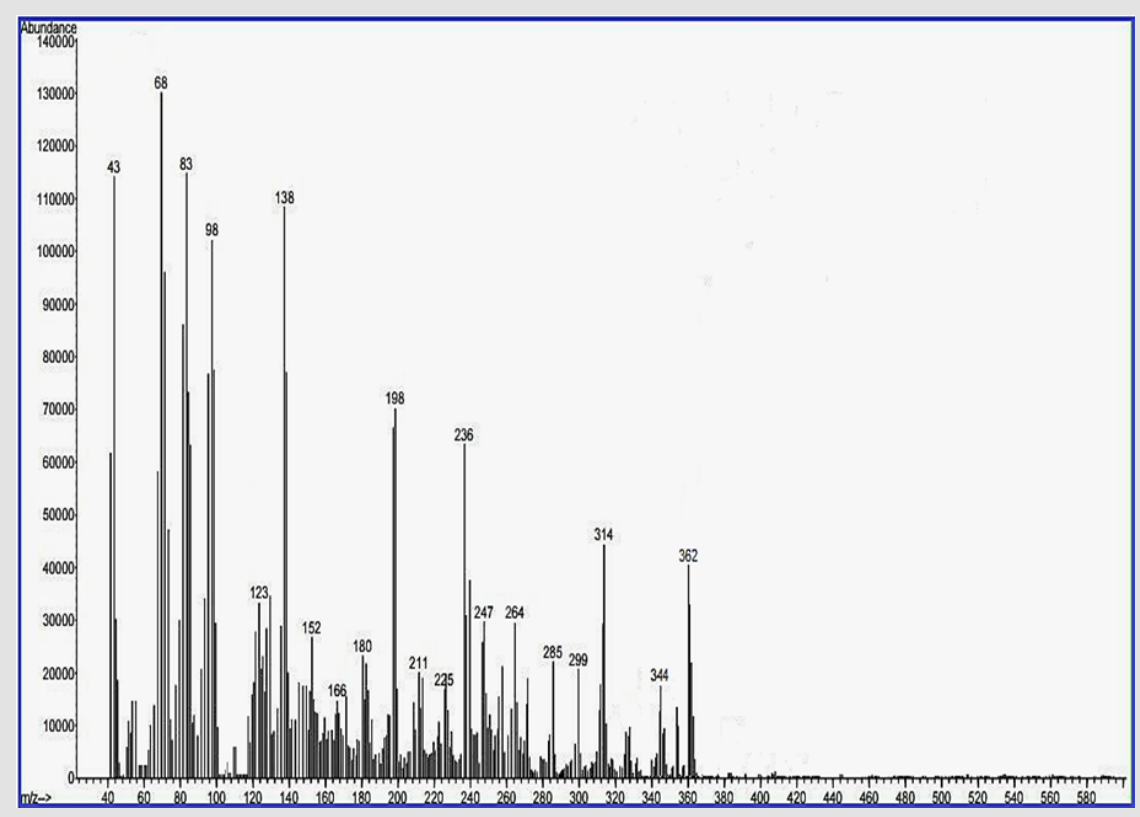

Figure 4: Mass-Spectrum of Invented Macrocyclic Sulfazan- Formazan Compound $\{3\}$. 


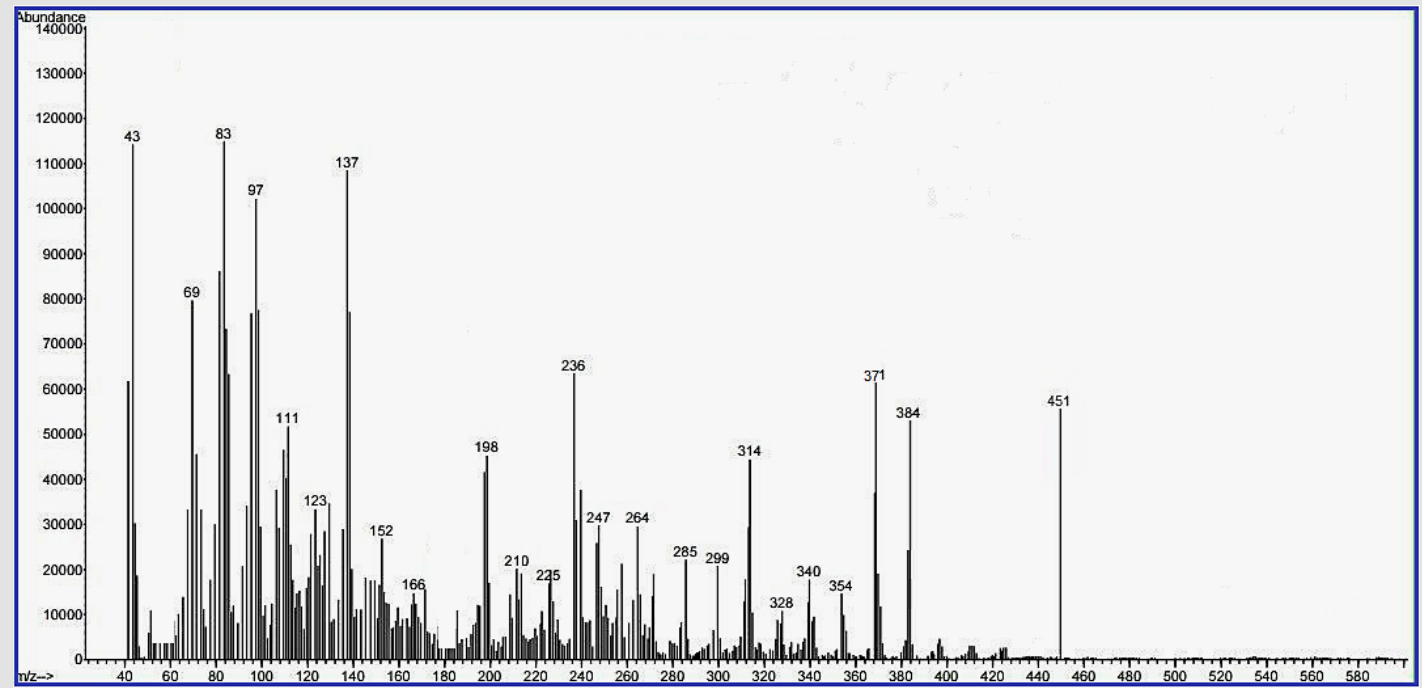

Figure 5: Mass-Spectrum of Invented Macrocyclic Sulfazan- Formazan of Triazole Compound $\{4\}$.

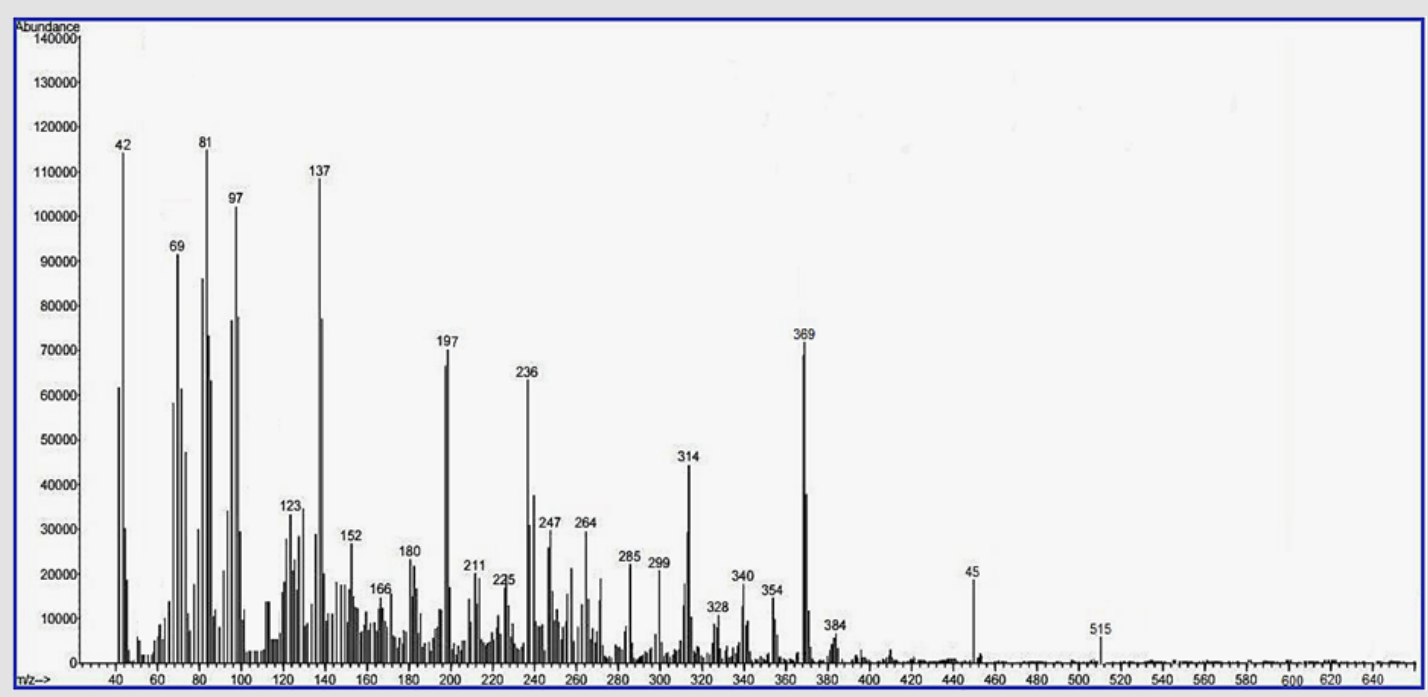

Figure 6: Mass-Spectrum of Invented Linear Sulfazan- Formazan Compound $\{5\}$.

\section{Other Characterization}

All Invented Macrocyclic Sulfazan- Formazan and Linear Sulfazan-Formazan were studied to collect all The chemical and physical properties, in Table 1.

\section{Antifungal Assay [17,18] of Invented Sulfazan-Formazan Derivatives}

The assessment of Invented Macrocyclic Sulfazan-Formazan derivatives and Invented Linear Sulfazan-Formazan derivatives tested against kinds of fungi epitomized through (Aspergillus) with (Candida Albicans) for all the invented compounds at three concentrations were occupied range of three analyses that occupied for each concentration $(20,30,50 \mu \mathrm{gm})$ rendering to the method [17,18], Table 2 . The results improved that the Invented Macrocyclic Sulfazan-Formazan compounds and Invented Linear Sulfazan- Formazan compounds have good results as inhibitor of fungi growth and the invented macrocyclic Sulfazan- Formazan compound [5] has more activity than other invented macrocyclic Sulfazan-formazan compound due to structure of compound [5] involved sufazan group (-N=N-S-) with formazan group (-N=C$\mathrm{N}=\mathrm{N}-$ ) more than other compounds besides to (Br) atoms, while compound [6] has less inhibition activity due to its structure ( $-\mathrm{OCH}_{3}$ ) methoxy group. 
Table 1: Other characterization of Invented Macrocyclic Formazan Compounds.

\begin{tabular}{|c|c|c|c|c|}
\hline Innovated Comps. & P \% & Color & M .P C & Rf \\
\hline Innovated Comp. $\{1\}$ & 86 & Yellowish & 164 & 0.66 \\
\hline Innovated Comp. $\{2\}$ & 82 & Deep Yellow & 192 & Ethanol : Dioxan \\
\hline Innovated Comp. $\{3\}$ & 84 & Yellowish Orange & 216 & Ethanol : Dioxan \\
\hline Innovated Comp. $\{4\}$ & 84 & Orange & 230 & 0.60 \\
\hline Innovated Comp. $\{5\}$ & 86 & Reddish Orange & 234 & Ethanol : Dioxan \\
\hline Innovated Comp. $\{6\}$ & 80 & Yellowish Orange & 222 & Ethanol : Dioxan \\
\hline
\end{tabular}

Table 2: Antifungal Assay of Invented Sulfazan-Formazan Compounds in Concentration ( $30 \mu . g m)$.

\begin{tabular}{|c|c|c|c|c|c|}
\hline Innovative Compounds & Aspergillus & Candida albicans & Innovative Compounds & Aspergillus & Candida albicans \\
\hline Innovated Comp. $\{1\}$ & + & + & Innovated Comp. $\{1\}$ & + & + \\
\hline Innovated Comp. $\{2\}$ & ++ & + & Innovated Comp. $\{2\}$ & ++ & + \\
\hline Innovated Comp. $\{3\}$ & ++ & ++ & Innovated Comp. $\{3\}$ & ++ & ++ \\
\hline Innovated Comp. $\{4\}$ & +++ & ++ & Innovated Comp. $\{4\}$ & +++ & ++ \\
\hline Innovated Comp. $\{5\}$ & +++ & +++ & Innovated Comp. $\{5\}$ & +++ & +++ \\
\hline Innovated Comp. $\{6\}$ & +++ & +++ & Innovated Comp. $\{6\}$ & +++ & +++ \\
\hline
\end{tabular}

Note:

$(+)$ : inhibition (4-7)mm

$(++)$ : inhibition (8-13)mm

$(+++)$ : inhibition (14-18)mm

\section{Conclusion}

All Invented Macrocyclic Sulfazan-Formazan derivatives and Invented Linear Sulfazan-Formazan compounds gave good evidences for their structures via various spectral techniques, also some of them studied against types of fungi that gave good data and good activity in inhibition of growth of Fungi.

\section{Conflict of Interest}

The authors declare that there is no conflict of interest.

\section{References}

1. Nagham Mahmood Aljamali, Imd Karm (2020) Development of Trimethoprim Drug and Innovation of Sulfazane-Trimethoprim Derivatives as Anticancer Agents. Biomedical \& Pharmacology Journal 13(2): 613-625.

2. Imd Karm, Hasaneen Kudhair Abdullabass, Nagham Mahmood Aljamali (2020) Invention of (Gluta.Sulfazane-Cefixime) Compounds as Inhibitors of Cancerous Tumors. Journal of Cardiovascular Disease Research 11(2): 44-55.

3. Nagham Mahmood Aljamali (2019) Review on (Azo, Formazane, Sulfazane)-Compounds. International Journal of Innovations in Scientific Engineering 10: 19-45.

4. Nagham Mahmood Aljamali (2021) Inventing of Macrocyclic Formazan Compounds and Studying Them Against Breast Cancer for The first Time Globally. Annals of pharma research 9(7): 525-533.

5. Nagham Mahmood Aljamali (2021) Synthesis Innovative Cyclic Formazan Compounds for The First Time and Evaluation of Their Biological Activity. International Journal Of Chemical And Lifesciences 10(11).
6. Nagham Mahmood Aljamali (2018) Experimental Methods for Preparation of Mannich Bases, Formazan, Normal and Cyclic Sulfur Compounds. In: Nagham Mahmood Aljamali (Edt.)., Evince pub Publishing House, India.

7. Nagham Mahmood Aljamali (2020) Alternative Methods in Organic Synthesis. In: Nagham Mahmood Aljamali (Edt.)., Eliva Press, Europe.

8. Nagham Mahmood Aljamali (2021) Effect of Conditions and Catalysis on Products. In: Nagham Mahmood Aljamali (Edt.)., Eliva Press, Europe.

9. Nagham Mahmood Aljamali (2019) The Various Preparation Methods in Synthetic Chemistry. In: Nagham Mahmood Aljamali (Edt.)., Evincepub Publishing house, India.

10. Nagham Mahmood Aljamali (2018) Reactions and Mechanisms. In: Nagham Mahmood Aljamali (Edt.)., IJMRA Publication.

11. Matheus ME, De Almeida Violante F, Garden SJ (2007) Isatins inhibit cyclooxygenase- 2 and inducible nitric oxide synthase in a mouse macrophage cell line. Eur J Pharmacol 556(1-3): 200-206.

12. Mid Mohmd, Nagham Mahmood Aljamali, Sabreen Ali Abdalrahman, Wassan Ala Shubber (2018) Formation of Oxadiazole Derivatives Ligands from Condensation and Imination Reaction with References To Spectral Investigation, Thermal and Microbial Assay. Biochem Cell Arch 18(1): 847-853.

13. Nagham Mahmood Aljamali (2016) Synthesis and Biological Study of Hetero (Atoms and Cycles) Compounds. Der Pharma Chemica 8(6): 4048.

14. Nagham Mahmood Aljamali (2015) Synthesis and Chemical Identification of Macro Compounds of (Thiazol and Imidazol). Research J Pharm and Tech 8(1): 78-84.

15. Mestaf M, Nawfel Muhammed Baqer Muhsin (2019) Treatment of (Electric Wires and Machines)- Erosion via Engineering Materials by the Coating. Neuro Quantology 17(11): 11-16. 
16. Nagham Mahmood Aljamali (2015) Review in Azo Compounds and its Biological Activity. Biochem Anal Biochem 4(2): 169.

17. Nagham Mahmood Aljamali, S F Jawad (2022) Preparation, Spectral Characterization, Thermal Study, and Antifungal Assay of (Formazane -Mefenamic acid)- Derivatives. Egypt J Chem 65(2): 449-457.

18. Nagham Mahmood Aljamali (2017) Synthesis of Antifungal Chemical Compounds from Fluconazole with (Pharma-Chemical) Studying. Research journal of Pharmaceutical, biological and chemical sciences 8(3): $564-573$

19. MN Abdmajed, Nagham Mahmood Aljamali (2021) Preparation of Benzothiazole-Formazane Reagents and Studying of (Spectral, Thermal, Scanning Microscopy, Biological Evaluation). International Journal of Pharmaceutical Research 13(1): 4290-4300.

20. Mad M, Nagham Mahmood Aljamali, Nadheema AA (2018) Preparation, Spectral Investigation, Thermal Analysis, Biochemical Studying of New (Oxadiazole -Five Membered Ring)-Ligands. Journal of Global Pharmacy Technology 10(1): 20-29.

21. Nagham Mahmood Aljamali (2020) Survey on Methods of Preparation and Cyclization of Heterocycles. International Journal of Chemical and Molecular Engineering 6(2): 19-36.

22. Mieaad M, Nagham Mahmood Aljamali, Wassan Ala Shubber, Sabreen Ali Abdalrahman (2018) New Azomethine- Azo Heterocyclic Ligands Via Cyclization of Ester. Research Journal of Pharmacy and Technology 11(6): 2555-2560.

23. Hasaneen Kudhair Abdullabass, Aseel Mahmood Jawad, Nagham Mahmood Aljamali (2020) Synthesis of drugs derivatives as inhibitors of cancerous cells. Biochem Cell Arch 20(2): 5315-5322.

24. Aseel Mahmood Jawad, Nagham Mahmood Aljamali (2020) Innovation, Preparation of Cephalexin Drug Derivatives and Studying of (Toxicity \& Resistance of Infection). International Journal of Psychosocial Rehabilitation 24(04): 3754-3767.

25. Hussein Ali Ahmed, Nagham Mahmood Aljamali (2021) Preparation, Characterization, Antibacterial Study, Toxicity Study of New Phenylene diamine- Formazan Derivatives. Indian Journal of Forensic Medicine \& Toxicology 15(2): 3102-3112.

26. Nagham Mahmood Aljamali, Hussein Mejbel Azeez (2021) Synthesis and Characterization of Some New Formazan - Cefixime and Study of Against Breast Cancer Cells. Annals of RSCB 25(4): 8562-8578.

27. Nagham Mahmood Aljamali, Asmaa Kefah Mahdi (2021) Synthesis, Identification and Anticancer Studying of Heterocyclic- Mefenamic Drug via Thiosemicarbazide. Annals of RSCB 25(4): 8521-8537.

28. S FJawad, Nagham Mahmood Aljamali (2021) Preparation, Investigation and Study of Biological Applications of Tyrosine Derivatives against Breast Cancer Cells . Neuro Quantology 19(9): 117-125.

29. Nagham Mahmood Aljamali, Intisar Obaid Alfatlawi (2015) Synthesis of Sulfur Heterocyclic Compounds and Study of Expected Biological Activity. Research J Pharm and Tech 8(9): 1225-1242.

ISSN: 2574-1241

DOI: 10.26717/BJSTR.2021.40.006453

Nagham Mahmood Aljamali. Biomed J Sci \& Tech Res

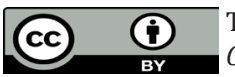

This work is licensed under Creative

Commons Attribution 4.0 License

Submission Link: https://biomedres.us/submit-manuscript.php
30. Nagham Mahmood Aljamali, Tabark Emad Al-Faham (2021) Synthesis, Identification, Chromatographic Studying of Formazane Phenylenediamine Derivatives. Annals of RSCB 25(4): 8538-8561.

31. Intisar Obaid Alfatlawi, Nuha SS, Zainab MJ ,Nagham Mahmood Aljamali (2017) Synthesis of New Organic Compounds Via Three Components Reaction with Studying of (Identification, Thermal Behavior, Bioactivity on Bacteria of Teeth. Journal of Global Pharma Technology 11(9): 157164.

32. Nagham Mahmood Aljamali, Saher Mahmood Jawd, Zainab MJ, Intisar Obaid Alfatlawi (2017) Inhibition activity of (Azo-acetyl acetone) on bacteria of mouth. Research Journal of Pharmacy and Technology 10(6): 1683-1686.

33. Nagham Mahmood Aljamali (2018) Synthesis, Investigation, Chromatography, Thermal)- Behavior of (Five, Seven)- Membered Ring with Azo and Anil Compounds. Pak J Biotechnol 15(1): 219-239.

34. Aseel Mahmood Jawad, Nagham Mahmood Aljamali, Saher Mahmood Jawd (2020) Development and Preparation of ciprofloxacin Drug Derivatives for Treatment of Microbial Contamination in Hospitals and Environment. Indian Journal of Forensic Medicine \& Toxicology 14(2): 1115-1122.

35. Nagham Mahmood Aljamali (2021) Spectral and Laboratory Diagnostics of Compounds. In: Nagham Mahmood Aljamali (Edt.)., Eliva Press, Europe.

36. Shireen R Rasool, Nagham Mahmood Aljamali, Ali Jassim Al-Zuhairi (2020) Guanine substituted heterocyclic derivatives as bioactive compounds. Biochem Cell Arch 20(2): 3651-3655.

37. Deniz Ar, Alexander FR Kilpatrick, Beatrice Cula, Christian Herwig, Christian Limberg (2021) Transformation of Formazanate at Nickel(II) Centers to Give a Singly Reduced Nickel Complex with Azoiminate Radical Ligands and Its Reactivity toward Dioxygen. Inorganic Chemistry 60(18): 13844-13853.

38. Francesca Milocco, Folkert de Vries, Harmke S Siebe, Silène Engbers, Serhiy Demeshko, et al. (2021) Widening the Window of Spin-Crossover Temperatures in Bis(formazanate)iron(II) Complexes via Steric and Noncovalent Interactions. Inorganic Chemistry 60(3): 2045-2055.

39. Abd Ali H, Nagham Mahmood Aljamali (2021) Chalcone-Heterocyclic Derivatives (Synthesis, Spectral Identification, Microbial Evaluation) ., International Journal of Pharmaceutical Research 13(1): 4234-4242.

40. Nor A, Sud S, Nagham Mahmood Aljamali (2019) Synthesis, Characterization and Thermal Analysis for New Amoxil Ligands. Asian Journal of Chemistry 31(5): 1022-1026.

41. Rajaa Abdul Ameer Ghafil, Nor A Alrab, Nagham Mahmood Aljamali (2020) Synthesis of Triazole Derivatives via Multi Components Reaction and Studying of (Organic Characterization, Chromatographic Behavior, Chem-Physical Properties). Egypt J Chem 63(11): 4163-4174.

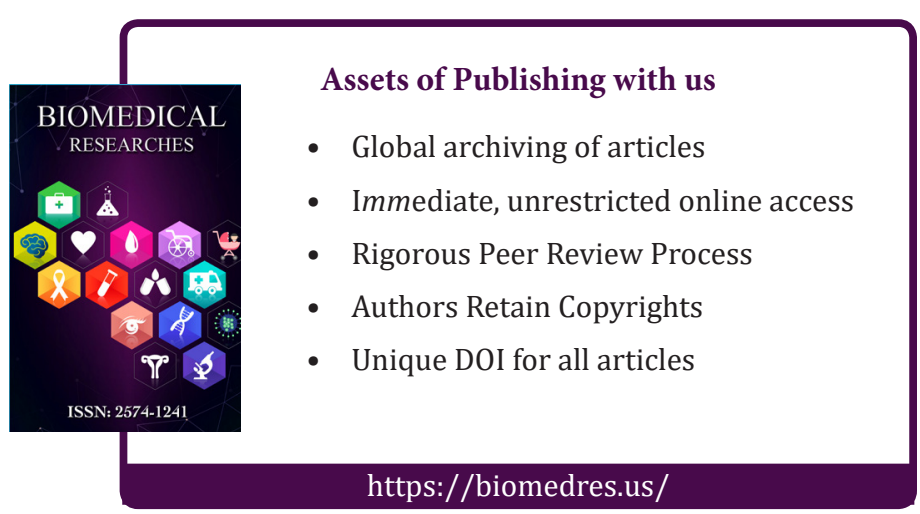

